\title{
Real-World Data on the Use of Hypertonic Saline Nasal Spray in ENT Practice
}

\author{
Christoph Bergmann ${ }^{1} \cdot$ Karolina Müller $^{2} \cdot$ Uta Thieme $^{3} \cdot$ Florian Zeman $^{2} \cdot$ Gunnar Huppertz $^{2} \cdot$ Michael Koller $^{2}$. \\ Peter Meiser ${ }^{4}(1)$
}

Accepted: 25 January 2019 / Published online: 9 February 2019

(C) The Author(s) 2019

\begin{abstract}
Hypertonic saline solutions are in broad medicinal use for treatment of various disorders of nose and paranasal sinuses. This trial aimed at the evaluation of safety and efficacy of a hypertonic saline nasal spray within the scope of routine care in an otorhinolaryngological outpatient setting. One hundred and thirty-six patients were included into this uncontrolled clinical trial and received 2.7\% saline solution nasal spray. Symptoms and clinical signs were documented on an ordinal scale of 0-4 during initial and final examination, and both individual symptoms and the sum of symptom scores (clinical sum score) were assessed. Participating patients and physicians furthermore evaluated the clinical benefit of the therapy. Clinical sum score decreased from $13.63 \pm 6.65$ to $7.85 \pm 6.79(p<0.001)$ between initial and final examination, and significant improvements were observed for 10 out of 12 individual symptoms. A clinical benefit was attributed to hypertonic saline treatment by both patients and physicians in more than $50 \%$ of the documented cases. Seventy percent of the patients noticed improvement of nasal breathing, and $50 \%$ of the patients considered hypertonic saline an alternative to the use of $\alpha$-sympathomimetics. One adverse event (epistaxis) was reported, whereas $95 \%$ of the patients rated the treatment as flawless. Eleven pregnant women and one nursing mother participated in the trial without the occurrence of any negative effect on mother and child. This study substantiates the safe and efficacious use of hypertonic saline nasal spray. Reduction in use of classical decongestants constitutes a valuable and safe therapeutic option for patients for whom use of these drugs is contraindicated.
\end{abstract}

Clinical Trial Register: DRKS-ID: DRKS00010825

Keywords Hypertonic saline $\cdot$ Nasal spray $\cdot$ Rhinitis $\cdot$ Rhinosinusitis $\cdot$ Decongestion $\cdot$ Pregnancy

\section{Introduction}

Saline nasal applications are broadly used in medicinal therapy. While isotonic saline solutions preferentially aim at cleansing and moistening of the nasal mucosa and thus are suitable for treatment of dry nose symptoms [1], hypertonic

This article is part of the Topical Collection on Medicine

Peter Meiser

p.meiser@ursapharm.de

ENT praxis Ruhr, Bocholder Straße 2, 45355 Essen, Germany

2 Center for Clinical Studies, University Hospital Regensburg, 93042 Regensburg, Germany

3 ENT praxis, Mercatorstraße 10-12, 47051 Duisburg, Germany

4 Ursapharm Arzneimittel GmbH, Industriestraße 35, 66129 Saarbruecken, Germany saline solutions are rather used for decongestion of the nasal mucosa. With increasing salt concentrations, the pure moistening effect is put in the rear and a water withdrawing effect comes to the fore, leading to decongestion of the affected tissues, an activity particularly wished in cases of viral, bacterial, or allergic rhinosinusitis [2-6]. Beyond this osmotic decongesting effect, removal of antigens and inflammatory mediators - thereby reducing the local inflammation - and improvement of mucociliar function may also contribute to relief from symptoms [7]. Dose-dependent adverse events due to hypertonicity (e.g., pain sensation, nasal blockage, rhinorrhea) have been demonstrated, occurring commonly when salt concentrations are above approximately $5 \%$. In contrast, therapeutic use of hypertonic saline exhibiting salt concentrations corresponding to less than $3 \%$ sodium chloride is generally recommended and well tolerable $[8,9]$. Decongestion by use of hypertonic saline is based on an osmotic effect, leading to the withdrawal of excess liquid from 
the edematous tissue. This non-pharmacological mode of action obviously leads to a less fast, less pronounced, and shorter lasting effect as compared to local application of $\alpha$ sympathomimetic drugs. $\alpha$-Sympathomimetics mimic the effects of agonists of the sympathetic nervous system on $\alpha 1$ and $\alpha 2$ receptors that lead immediately to constriction of the nasal blood vessels and consequently to nasal decongestion. On the other hand, $\alpha$-sympathomimetics should not be used for a prolonged time (due to local side effects including rebound reactions and the occurrence of dry nose symptoms) and only after careful risk-benefit assessment in, among others, pregnant and lactating women and in patients suffering from cardiovascular diseases. Application of hypertonic saline therefore constitutes an alternative or adjunct treatment to the use of $\alpha$-sympathomimetic nasal sprays for these populations.

Besides long-standing use of large-volume saline nasal rinsings that are particularly suitable for cleaning of the nasal mucosa, supporting free nasal breathing by softening and dislodging the mucus lining of the nasal cavity, the number of decongesting hypertonic saline nasal spray products increased steadily in the recent years [10]. However, only few data are available on the use of hypertonic saline nasal spray formulations. We therefore initiated a prospective clinical trial according to $\S 23 \mathrm{~b}$ of the German Medical Device Act in order to assess the clinical benefits of a treatment with a commercially available hypertonic saline nasal spray. No exclusion criteria were prespecified beyond the information given in the Instruction For Use. Clinical investigations according to $\S 23 \mathrm{~b}$ of the German Medical Device Act are performed with CE-marked, thus marketed, medical devices, must be conducted within the intended use of the device and without the application of any additional invasive or otherwise stressful examination. By using this design collection of efficacy and safety data in differing indications, periods of treatment and also in special populations such as young children, geriatric patients, and pregnant and lactating women were possible within the scope of routine care in an otorhinolaryngological outpatient setting.

\section{Materials and Methods}

\section{Trial Design}

This clinical trial was based on a prospective, observational, longitudinal design with two points of measurement, initial and final exams. The trial was conducted according to $\S 23 \mathrm{~b}$ of the German Medical Device Act in patients that had an indication for application of hypertonic saline nasal sprays, including but not limited to, e.g., viral, bacterial, or allergic rhinitis, acute or chronic rhinosinusitis, and use as an alternative to $\alpha$-sympathomimetics.
The study was carried out in two trial centers in Germany during the period May 2016-August 2017. Patients included in the study were supposed to be above the age of 1 year or older. No further inclusion or exclusion criteria were defined beyond the information given in the Instruction For Use. However, the decision to prescribe/recommend hypertonic saline nasal spray to the respective patient must have been made by the physician before the inclusion of the patient in the trial, and patients were enrolled only after they had been given sufficient information and had provided written consent concerning their participation in the trial. Each patient enrolled was subject to an initial and a final examination. Due to the non-interventional character of the study, the time course of the visits was not prespecified.

The trial was performed according to the German Medical Device Act and the Declaration of Helsinki, was registered in the German Clinical Trial Register (DRKS-ID: DRKS00010825), and was approved by the Institutional Review Board of the Freiburg Ethics Commission International (Code: 016/1245).

\section{Trial Product}

The medical device used in this trial was manufactured at Ursapharm Arzneimittel GmbH, Saarbruecken, Germany. The hypertonic saline nasal spray (hysan® Salinspray) contains $2.7 \%$ sea salt and purified water. Patients were advised to apply the nasal spray several times a day while spraying one to two sprays into each nostril, in compliance to the manufacturer's Instruction For Use. With each spray, $0.07 \mathrm{~mL}$ of saline solution was dispensed. In case of concomitant treatment with other nasal sprays or nasal drops, patients were advised to comply with a time interval of at least $30 \mathrm{~min}$ between the applications. In case of the occurrence of hypersensitivity reactions like itching or redness, patients were advised to discontinue use of the product.

\section{Efficacy and Safety Measurements}

All data for this study were recorded on a single case report form (CRF) that was filled in by the physician. Indications for application of hypertonic saline nasal sprays were documented at the initial examination, and symptoms and clinical signs were subsequently documented at both visits (initial and final examination). Impairment of nasal breathing/nasal obstruction, sneezing attacks, nasal discharge anterior/runny nose, nasal discharge posterior, thick nasal discharge, desire to clear one's throat/dry throat, redness of nasal mucosa, edema of nasal mucosa, impairment of smell, impairment of taste, impairment of sleep, and impairment of food intake were assessed on an ordinal scale of $0-4(0=$ none, $1=$ mild, $2=$ moderate, $3=$ strong, $4=$ very strong), and the individual ratings were documented for each symptom and each visit. 
Redness of nasal mucosa and edema of nasal mucosa were determined by anterior rhinoscopy; all other parameters assessed were reported by the patients. Scorings of each symptom and clinical sign were summed up to the clinical sum score ranging from 0 (no symptoms) to 48 (very strong symptoms). One hundred and twenty-nine patients had complete ratings of the clinical sum score at both visits, and missings were not imputed.

In order to evaluate the clinical benefit of the hypertonic saline nasal spray therapy, the physicians involved were also asked to assess the treatment with the product (very goodgood-satisfactory-bad), the compliance of the patient (very good-good-satisfactory-bad), whether treatment with hypertonic saline should be continued (yes-no), how they assessed the period until the resumption of activities of daily living as compared to treatment without hypertonic saline (significantly shorter-shorter-no difference-longer-significantly longer), and whether they would prescribe/recommend the product again under similar conditions (yes-no-possibly). During the final examination, the physicians involved also evaluated the use of $\alpha$-sympathomimetics by the patients as reducedconstant-increased or not assessable.

During the final examination, the patients evaluated the overall treatment effect (significantly better-better-no difference-worse-significantly worse), their satisfaction with hypertonic saline nasal spray (very good-good-satisfactorybad), whether they would use it again under similar conditions (yes-no), when they experienced an improvement of nasal breathing after application of hypertonic saline nasal spray ( $<2 \mathrm{~min},<5 \mathrm{~min},<10 \mathrm{~min},<30 \mathrm{~min}$, after repeated application, no improvement), and whether they consider hypertonic saline nasal sprays an alternative option for use of $\alpha$ sympathomimetics (yes-no-no alternative, but adjunct treatment).

Tolerability of the product was evaluated by the physicians at the final examination using the ratings "flawless," "acceptable," and "not acceptable." Adverse events and severe adverse events had to be documented in the case report form at every visit by the investigator. The occurrence of severe adverse events had to be reported to the sponsor of the study within $24 \mathrm{~h}$.

\section{Sample Size and Statistical Analysis}

This clinical trial was designed to provide real-world evidence by means of a number of clinically relevant end points. Thus, no primary end point for formal sample size calculation was defined. According to a pragmatic sample size rationale, the inclusion of a maximum of 150 patients was planned to obtain valid and reliable results regarding safety and efficacy. Patient recruitment took place between May 2016 and August 2017.

Data are presented as mean $(\mathrm{m}) \pm$ standard deviation (SD) for continuous variables or as relative frequencies $(n)$ and percentages $(\%)$ for categorical variables. Occasionally, percentages did not add up to $100 \%$ due to rounding. MannWhitney $U$ test was used to compare the interval between initial and final examination between patients with single diagnosis of acute or chronic rhinosinusitis. Clinical sum scores as well as single symptoms and clinical signs between initial and final examination were compared using Wilcoxon signedrank tests. Moreover, Kruskal-Wallis $H$ tests were used to compare changes in single symptoms and clinical signs (categorized as improvement, no change, worsening) for a segment of patients with a clearly defined indication (either acute rhinosinusitis, chronic rhinosinusitis, allergic rhinitis, nonallergic rhinitis, or alternative to use of $\alpha$-sympathomimetics, $n=103$ ). A $p$ value of $\leq 0.05$ was considered as statistically significant. Statistical analyses were explorative. Thus, no adjustments for multiple testing were performed. Normal distribution of metric variables was assessed by Shapiro-Wilk test. All analyses were performed using SAS 9.4 (SAS Institute Inc., Cary, NC, USA) and SPSS, version 25 (IBM Deutschland GmbH, Germany).

\section{Results}

A total of 136 patients (43 males, 93 females) were included in the study (first patient in: May 24, 2016; last patient out: August 7, 2017). Three patients were lost to follow-up; thus, the ITT population included $n=136$ patients at baseline and $n=133$ at the final examination. The safety population comprised all patients included into the study $(n=136)$ (Fig. 1). On average, the final examination took place $44.96( \pm$ 51.14) days after the initial examination. The time between initial and final examination was significantly shorter in patients with acute rhinosinusitis (33.71 \pm 38.05 days) than in patients with chronic rhinosinusitis $(78.00 \pm 98.68$ days; $\left.U_{(31,14)}=315, p=0.016\right)$.

Overall, compliance was assessed by the physicians as "very good" in 58\%, as "good" in $37 \%$, as "satisfactory" in $4 \%$, and as "bad" in $1.5 \%$ of the patients.

Demographic data and indications for use of hypertonic saline nasal spray are given in Tables 1 and 2 .

\section{Efficacy End points}

Clinical sum scores (sum of symptom scores for impairment of nasal breathing/nasal obstruction, sneezing attacks, nasal discharge anterior/runny nose, nasal discharge posterior, thick nasal discharge, desire to clear one's throat/dry throat, redness of nasal mucosa, edema of nasal mucosa, impairment of smell, impairment of taste, impairment of sleep, and impairment of food intake) for the initial and final examinations are given in Fig. 2. Clinical sum score decreased significantly from 13.63 


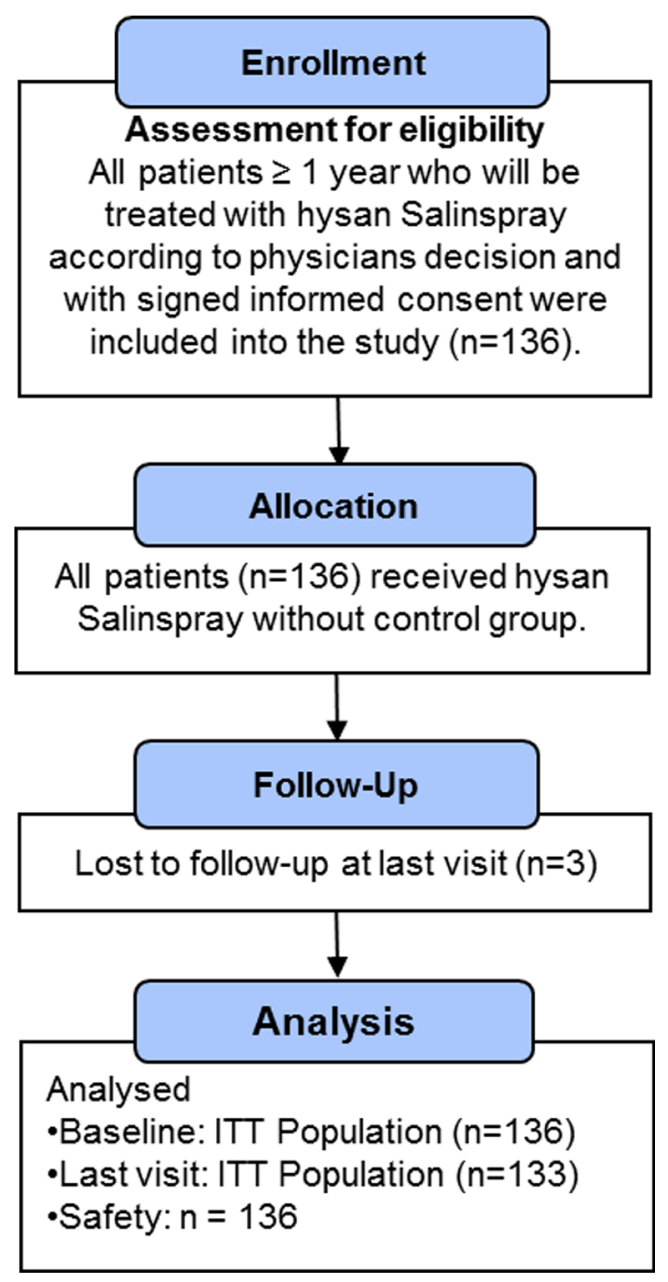

Fig. 1 CONSORT flow diagram

\pm 6.65 to $7.85 \pm 6.79$ score points $(z=-8.06, p<0.001, n=$ $129)$ between initial and final examination.

Ratings for each symptom revealed statistically significant reductions in 10 out of 12 symptoms $(p<0.01)$, only for parameters "impairment of taste" and "impairment of food intake" no significant change in symptoms was observed $(p>0.05)$. However, large part of the study population did not express any symptoms already at initial examination for the latter two parameters (Table 3).

Table 1 Baseline characteristics of included patients

\begin{tabular}{lccc}
\hline & Number & Mean $( \pm$ st.dev. $)$ & Median (min-max) \\
\hline Sex & & & \\
$\quad$ Male & 43 & & \\
Female & 93 & & $42.62(5-86)$ \\
Age (years) & 136 & $44.16 \pm 18.99$ & $169.00(110.00-193.00)$ \\
Height $(\mathrm{cm})$ & 136 & $169.53 \pm 10.08$ & $74(20.00-147.00)$ \\
Weight $(\mathrm{kg})$ & 136 & $75.58 \pm 18.19$ & $25.69(14.38-50.87)$ \\
BMI $\left(\mathrm{kg} / \mathrm{m}^{2}\right)$ & 136 & $26.11 \pm 5.31$ & \\
\hline
\end{tabular}

Table 2 Indications for use of hypertonic saline nasal spray

Number

\begin{tabular}{lc} 
Indication $^{\text {a }}$ & \\
Acute rhinosinusitis & 36 \\
Chronic rhinosinusitis & 17 \\
Allergic rhinitis & 14 \\
Non-allergic rhinitis & 16 \\
Alternative to use of $\alpha$-sympathomimetics & \\
$\quad$ Rhinitis medicamentosa & 13 \\
Irritated nasal mucous membrane & 29 \\
Other indications & \\
Nasal obstruction & 13 \\
$\quad$ Nasal concha hyperplasia & 6 \\
Hyposmia & 1 \\
Rhinitis sicca & 4 \\
Chronic rhinitis & 1 \\
Serous otitis media & 1 \\
Eustachian catarrh & 3 \\
Septum perforation & 1 \\
\hline
\end{tabular}

${ }^{\text {a }}$ Multiple answers possible

At the final examination, physicians involved assessed the treatment with the product as very good $(22 \%)$, good $(35 \%)$, satisfactory (35\%), or bad (8\%). The treatment should be continued in $42 \%$ of the cases (e.g., due to underlying chronical disease); in $58 \%$ of the cases, treatment with hypertonic saline was ceased after the final examination. Of the physicians, $4.5 \%$ assessed the period until the resumption of activities of daily living as compared to a treatment without hypertonic saline as significantly shorter, $41 \%$ as shorter; in $53 \%$ of the cases, no difference was observed; and in $0.75 \%$, a longer period was observed. Physicians would prescribe hypertonic saline again in patients with identical diagnoses in $57 \%$ of the cases, would not prescribe it in $26 \%$, and would possibly prescribe it in $17 \%$ of the cases. Use of $\alpha$-sympathomimetics was assessed by the physicians as reduced in $34 \%$ of the patients, as constant in $35 \%$, and as increased in $1.5 \%$ of the cases. In $29 \%$ of the cases, use of $\alpha$-sympathomimetics by the patients was rated as not assessable.

Thirteen percent of the patients felt significantly better during treatment, $45 \%$ rated their condition as better, $38 \%$ recognized no difference, $3 \%$ rated their condition as worse, and $0.75 \%$ as significantly worse. Satisfaction with hypertonic saline nasal spray was assessed by the patients as very good in $16 \%$ of the cases, as good in $38 \%$, as satisfactory in $36 \%$, and as bad in $10 \%$ of the cases. Sixty-one percent of the patients would use hypertonic saline nasal spray again under similar conditions, and $38 \%$ would not.

Fifty percent of the patients considered hypertonic saline nasal sprays as an alternative option for use of $\alpha$-sympathomimetics, whereas $30 \%$ of the patients did not. Fourteen of the 


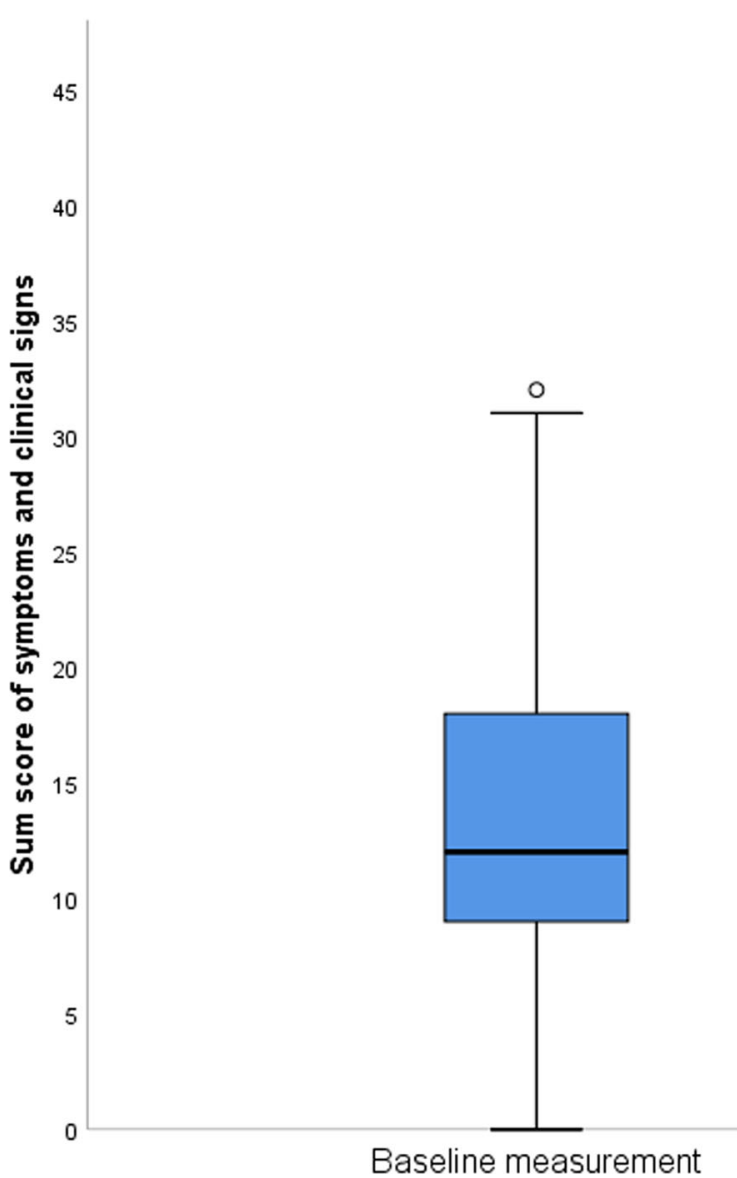

Fig. 2 Boxplot of clinical sum scores for initial and final examination, representing the sum of symptom scores for impairment of nasal breathing/nasal obstruction, sneezing attacks, nasal discharge anterior/ runny nose, nasal discharge posterior, thick nasal discharge, desire to

patients rated hypertonic saline nasal sprays not as an alternative but as an adjunct treatment to the use of $\alpha$ sympathomimetics (6\% missing values).

Improvement of nasal breathing after application of hypertonic saline nasal spray was observed in ca. $70 \%$ of the patients. Table 4 gives an overview on the onset of improvement as observed by the patients.

In addition, we compared symptoms and clinical signs for specific subgroups of patients. We considered patients who received the hypertonic saline spray for either one of the five specific indications: acute rhinosinusitis, chronic rhinosinusitis, allergic rhinitis, non-allergic rhinitis, and alternative to use of $\alpha$-sympathomimetics ( $n=103$ ); patients with double indications or "other indications" were omitted from these analyses $(n=33)$. No significant differences in changes in single symptoms and clinical signs between these specific patient groups were observed for 11 out of 12 parameters tested. A significant difference was found for parameter "nasal discharge, posterior" $(p=0.011)$. Patients suffering from "chronic rhinosinusits" were more likely to show improvements $(71 \%$ improvement vs. $21 \%$ no change vs. $7 \%$ worsening) in parameter "nasal discharge, posterior" as compared to patients suffering from "acute rhinosinusitis" (29\% improvement, $65 \%$ no change, $6 \%$ worsening) and using the nasal spray as "alternative to use of $\alpha$-sympathomimetics" ( $15 \%$ improvement, $64 \%$ no change, $21 \%$ worsening).

\section{Safety Evaluation}

Tolerability was assessed as "flawless" in $95 \%$ of the patients and as "acceptable" in 5\% of the applications. One patient reported twice about the occurrence of an epistaxis, which was assessed by the physician as being "possibly related" to the application of the nasal spray.

Eleven patients were pregnant during application of the hypertonic saline nasal spray, and one patient was breastfeeding. In none of these patients, any adverse events were reported. Follow-up of the pregnancy, thus monitoring of the health status of the newborn, was conducted in 10 cases (one case lost to follow-up; follow-up during ongoing visits or via telephone). Health status of the nursing child was also monitored. No signals on a negative effect on health of the 
Table 3 Baseline and outcome ratings for initial and final examination for all individual symptoms assessed

\begin{tabular}{|c|c|c|c|c|c|c|c|c|}
\hline & Baseline & Outcome & Baseline & Outcome & Baseline & Outcome & Baseline & Outcome \\
\hline & \multicolumn{2}{|c|}{ Impairment of nasal breathing/nasal obstruction } & \multicolumn{2}{|c|}{ Sneezing attacks } & \multicolumn{2}{|c|}{ Nasal discharge, anterior/runny nose } & \multicolumn{2}{|c|}{ Nasal discharge, posterior } \\
\hline None & $20(15.5)$ & $50(38.8)$ & $53(41.1)$ & $75(58.1)$ & $70(54.3)$ & $81(62.8)$ & $62(48.1)$ & $78(60.5)$ \\
\hline Slight & $25(19.4)$ & $39(30.2)$ & $36(27.9)$ & $36(27.9)$ & $19(14.7)$ & $27(20.9)$ & 24 (18.6) & $21(16.3)$ \\
\hline Moderate & $45(34.9)$ & $26(20.2)$ & $26(20.2)$ & $13(10.1)$ & $22(17.1)$ & $13(10.1)$ & $29(22.5)$ & $22(17.1)$ \\
\hline Strong & $32(24.8)$ & $13(10.1)$ & $12(9.3)$ & $5(3.9)$ & $10(7.8)$ & $7(5.4)$ & $14(10.9)$ & $8(6.2)$ \\
\hline Very strong & \multirow{2}{*}{\multicolumn{2}{|c|}{$<0.001$}} & $2(1.6)$ & $0(0)$ & $8(6.2)$ & $1(0.8)$ & $0(0)$ & $0(0)$ \\
\hline$p$ value & & & \multicolumn{2}{|c|}{$<0.001$} & \multicolumn{2}{|c|}{$<0.001$} & \multicolumn{2}{|c|}{0.001} \\
\hline & \multicolumn{2}{|l|}{ Thick nasal discharge } & \multicolumn{2}{|c|}{ Desire to clear one's throat/dry throat } & \multicolumn{2}{|c|}{ Redness of nasal mucosa } & \multicolumn{2}{|c|}{ Edema of nasal mucosa } \\
\hline None & $75(58.1)$ & $93(72.1)$ & $50(38.8)$ & $71(55.0)$ & $8(6.2)$ & $43(33.3)$ & $14(10.9)$ & $48(37.2)$ \\
\hline Slight & $20(15.5)$ & $18(14.0)$ & $28(21.7)$ & $26(20.2)$ & $40(31.0)$ & $65(50.4)$ & $16(12.4)$ & $56(43.4)$ \\
\hline Moderate & $21(16.3)$ & $11(8.5)$ & $29(22.5)$ & $18(14.0)$ & $68(52.7)$ & $16(12.4)$ & $72(55.8)$ & $22(17.1)$ \\
\hline Strong & $11(8.5)$ & $6(4.7)$ & $17(13.2)$ & $12(9.3)$ & $13(10.1)$ & $4(3.1)$ & $26(20.2)$ & $3(2.3)$ \\
\hline Very strong & \multirow{2}{*}{\multicolumn{2}{|c|}{0.002}} & $5(3.9)$ & $2(1.6)$ & $0(0)$ & $1(0.8)$ & $1(0.8)$ & $0(0)$ \\
\hline$p$ value & & & \multicolumn{2}{|c|}{$<0.001$} & \multicolumn{2}{|c|}{$<0.001$} & \multicolumn{2}{|c|}{$<0.001$} \\
\hline & \multicolumn{2}{|l|}{ Impairment of smell } & \multicolumn{2}{|c|}{ Impairment of taste } & \multicolumn{2}{|c|}{ Impairment of sleep } & \multicolumn{2}{|c|}{ Impairment of food intake } \\
\hline None & $71(55.0)$ & $86(66.7)$ & $91(70.5)$ & $101(78.3)$ & $50(38.8)$ & $84(65.1)$ & $107(82.9)$ & $117(90.7)$ \\
\hline Slight & $18(14.0)$ & $19(14.7)$ & 15 (11.6) & $12(9.3)$ & $19(14.7)$ & $16(12.4)$ & $12(9.3)$ & $6(4.7)$ \\
\hline Moderate & $20(15.5)$ & 15 (11.6) & $13(10.1)$ & $9(7.0)$ & $23(17.8)$ & $17(13.2)$ & $7(5.4)$ & $4(3.1)$ \\
\hline Strong & $14(10.9)$ & $5(3.9)$ & $5(3.9)$ & $2(1.6)$ & $27(20.9)$ & $9(7.0)$ & $3(2.3)$ & $2(1.6)$ \\
\hline Very strong & $6(4.7)$ & $4(3.1)$ & \multirow{2}{*}{\multicolumn{2}{|c|}{$0.083^{5(3.9)}$}} & \multirow{2}{*}{\multicolumn{2}{|c|}{$<0.001$}} & $0(0)$ & $0(0)$ \\
\hline$p$ value & \multicolumn{2}{|l|}{0.002} & & & & & \multicolumn{2}{|c|}{0.099} \\
\hline
\end{tabular}

Absolute numbers are given for each parameter and rating; relative percentages are given in brackets

unborn/newborn children after application of hypertonic saline nasal spray to the patients were observed.

\section{Discussion}

The nasal mucous membrane reacts in a comparable manner on diverse stimuli such as infection, allergy, or other trigger factors with inflammation, edema, and the production of aqueous or sticky hypersecretions. Various clinical symptoms result including impairment of nasal breathing, a runny nose, and sneezing attacks that are commonly observed in different disease entities [3, 4]. Even though many of these diseases, in particular acute viral rhinosinusitis, are self-limiting, patients regularly seek medical assistance from doctors and pharmacists in order to relieve their complaints. $\alpha$-Sympathomimetics are most frequently applied in order to decrease congestion and thereby to restore free nasal breathing. Topical decongestion of inferior and

Table 4 Onset of improvement of nasal breathing after application of hypertonic saline nasal spray

\begin{tabular}{lll}
\hline Onset of improvement & \% of patients & Cumulative \% \\
\hline$<2$ min & 16 & 16 \\
$<5 \mathrm{~min}$ & 16 & 32 \\
$<10 \mathrm{~min}$ & 13 & 45 \\
$<30 \mathrm{~min}$ & 10 & 55 \\
After repeated application & 14 & 69 \\
No improvement & 29 & \\
\hline
\end{tabular}

middle turbinates and infundibular mucosa was demonstrated; clinical benefits beyond decongestion of these inner structures of the nose, such as sinus and Eustachian tube ventilation and drainage, remain to be demonstrated [11, 12]. Furthermore, vasoconstriction induced by nasal decongestants may be followed by rebound congestion and longlasting alterations of the nasal mucosa $[13,14]$; therefore, these drugs should not be used for longer than 1 week, and special populations such as pregnant and breast-feeding women should use $\alpha$-sympathomimetics only after careful risk-benefit-assessment [15].

Hypertonic saline nasal sprays might therefore constitute alternative or at least adjunct treatments to the use of pharmacologically acting decongestants. Beyond the decongesting effect that is achieved through the osmotic action of the solution, saline solutions are commonly used for mechanical cleansing and moistening of the nasal mucosa. Moreover, promotion of mucociliary clearance was described for hypertonic saline solutions [16].

The aim of this trial was to evaluate the efficacy and safety of a hypertonic saline nasal spray on various diseases of nose and paranasal sinuses. Some of the indications for use of the nasal spray (given in Table 2) might be considered as uncommon, such as use in case of hyposmia, or even as rather counterproductive, as would be the case for use in rhinitis sicca. Due to cooccurrence of disease states, however, such as dry nose symptoms due to an underlying rhinitis medicamentosa [1], collection of such practice-related data remains important. Established indications for use of saline irrigations such as acute and chronic rhinosinusitis as well as allergic rhinitis constitute the main focus of this study, however [4-6]. 
The assessment of various disease states under hypertonic saline treatment furthermore allowed us to tease out a more pronounced effect for "rhinosinusitis acute/chronic" vs. "alternative to use of $\alpha$-sympathomimetics" with regard to parameter "nasal discharge, posterior," which is certainly due to the frequent occurrence of this symptom in patients suffering from rhinosinusitis on the one hand, but which also can be attributed to a bettering of the overall clinical presentation of these patients. The clinical sum score expressing combined severity of 12 symptoms decreased significantly between initial and final examination during treatment with hypertonic saline nasal spray. Due to potential self-limitations of clinical symptoms, the observed effects were also evaluated for clinical relevance by the included physicians and patients. For more than half of the documented cases, both patients and physicians reported an overall positive effect of the treatment and thus would take or recommend the hypertonic saline spray again under similar conditions, respectively. Approximately $70 \%$ of the patients observed an improvement of nasal breathing, several even after a very short period of time as one third of the patients noticed improvement already after 2 or $5 \mathrm{~min}$ after application. To our knowledge, this is a first detailed report on the onset of the clinical effect of hypertonic saline nasal spray in routine clinical practice, replicating and extending results of an earlier trial applying rhinomanometry [17].

Concomitant treatments were allowed during the conduct of the study in order to maintain the non-interventional character and to evaluate the potential additional benefit from therapy with hypertonic saline. A clear reduction or even abstention from use of $\alpha$-sympathomimetics must be highlighted as an important finding of this trial. In particular, patients for which use of these drugs is unsuitable - such as pregnant and nursing women, people suffering from irritated and dry nasal mucosa, or patients having a history of abuse of decongesting nasal sprays that search for nonpharmacological therapeutic options - may profit from treatment with this simple osmotic principle.

Furthermore, our trial confirms the safety of therapy with hypertonic saline. Only one patient reported about the occurrence of epistaxis that was rated as potentially related to the application of the nasal spray, and none out of 11 pregnant patients and one patient with a nursing child reported any negative effects. Thus, the $2.7 \%$ saline solution nasal spray application showed an overall good risk-benefit ratio which is in accordance with previous findings [18-22]. Male patients were underrepresented in this trial, and this was also observed in a similar study investigating daily hypertonic saline nasal irrigation among patients with sinusitis [23]. Some of the saline applied will be ingested by the patients, which should be considered in populations that should limit their salt consume. Due to the overall low volume applied with the tested nasal spray, this amount can be neglected, however.
Obviously, the observed results must be reflected against the self-limiting course of symptoms for which nasal sprays are used and the uncontrolled study design. The use of both patient and physician assessments, in the biometric calculation and interpretation of the clinical benefit, compensates this drawback in design to a certain extent. In conclusion, the present findings replicate clinical data from controlled studies on illustrating the overall safety and efficacy of various nasal hypertonic saline applications [19, 24-29].

\section{Compliance with Ethical Standards}

The trial was performed according to the German Medical Device Act and the Declaration of Helsinki, was registered in the German Clinical Trial Register (DRKS-ID: DRKS00010825), and was approved by the Institutional Review Board of the Freiburg Ethics Commission International (Code: 016/1245).

Conflict of Interest The trial was funded by URSAPHARM Arzneimittel GmbH. Peter Meiser is employed at URSAPHARM Arzneimittel $\mathrm{GmbH}$. The authors have declared that there is no further conflict of interest.

Research Involving Human Participants All procedures performed in studies involving human participants were in accordance with the ethical standards of the institutional research committee and with the 1964 Helsinki declaration and its later amendments or comparable ethical standards.

Informed Consent Informed consent was obtained from all individual participants included in the study.

Open Access This article is distributed under the terms of the Creative Commons Attribution 4.0 International License (http:// creativecommons.org/licenses/by/4.0/), which permits unrestricted use, distribution, and reproduction in any medium, provided you give appropriate credit to the original author(s) and the source, provide a link to the Creative Commons license, and indicate if changes were made.

Publisher's Note Springer Nature remains neutral with regard to jurisdictional claims in published maps and institutional affiliations.

\section{References}

1. Hildenbrand T, Weber RK, Brehmer D. Rhinitis sicca, dry nose and atrophic rhinitis: a review of the literature. Eur Arch Otorhinolaryngol. 2011;268(1):17-26.

2. Miwa M, Nakajima N, Matsunaga M, Watanabe K. Measurement of water loss in human nasal mucosa. Am J Rhinol. 2006;20(5): $453-5$.

3. Fokkens W, Lund V, Mullol J, Group obotEPPoRaNP. European position paper on rhinosinusitis and nasal polyps. Rhinology. 2007;20(Suppl):1-136.

4. Fokkens WJ, Lund VJ, Mullol J, Bachert C, Alobid I, Baroody F, et al. EPOS 2012: European position paper on rhinosinusitis and nasal polyps 2012. A summary for otorhinolaryngologists. Rhinology. 2012;50(1):1-12. https://doi.org/10.4193/Rhino50E2.

5. Head K, Snidvongs K, Glew S, Scadding G, Schilder AG, Philpott $\mathrm{C}$, et al. Saline irrigation for allergic rhinitis. Cochrane Database 
Syst Rev. 2018;6:Cd012597. https://doi.org/10.1002/14651858. CD012597.pub2.

6. Chong LY, Head K, Hopkins C, Philpott C, Glew S, Scadding G, et al. Saline irrigation for chronic rhinosinusitis. Cochrane Database Syst Rev. 2016;4:Cd011995. https://doi.org/10.1002/14651858. CD011995.pub2.

7. Brown C, Graham S. Nasal irrigations: good or bad? Curr Opin Otolaryngol Head Neck Surg. 2004;12(1):9-13.

8. Principi N, Esposito S. Nasal irrigation: an imprecisely defined medical procedure. Int J Environ Res Public Health. 2017;14(5).

9. Baraniuk J, Ali M, Yuta A, Fang S, Naranch K. Hypertonic saline nasal provocation stimulates nociceptive nerves, substance $\mathrm{P}$ release, and glandular Mucous exocytosis in normal humans. Am J Respir Crit Care Med. 1999;160(2):655-62.

10. Insight-Health. Over-the-counter sales data (Pharmacies, Germany). Apo-Channel 2013-2018

11. Stringer S, Mancuso A, Avino A. Effect of a topical vasoconstrictor on computed tomography of paranasal sinus disease. Laryngoscope. 1993;103(1 Pt 1):6-9.

12. Benammar-Englmaier M, Hallermeier J, Englmaier B. Alphamimetische Wirkungen an der Nasenschleimhaut im MRTomogramm. Digit Bilddiagn. 1990;10(46-50)

13. Snidvongs $\mathrm{K}$, Thanaviratananich $\mathrm{S}$. Update on intranasal medications in rhinosinusitis. Curr Allergy Asthma Rep. 2017;17(7):0170720 .

14. Ramey JT, Bailen E, Lockey RF. Rhinitis medicamentosa. J Investig Allergol Clin Immunol. 2006;16(3):148-55.

15. Mazzotta P, Loebstein R, Koren G. Treating allergic rhinitis in pregnancy. Safety considerations. Drug Safety. 1999;20(4):361-75.

16. Homer J, Dowley A, Condon L, El-Jassar P, Sood S. The effect of hypertonicity on nasal mucocilliary clearance. Clin Otolaryngol Allied Sci. 2000;25(6):558-60.

17. Michel O, Dressler A. Hypertonic (3\%) vs. isotonic brine nosespray-a controlled study. Laryngorhinootologie. 2011;90(4):206-10.

18. Chirico G, Quartarone G, Mallefet P. Nasal congestion in infants and children: a literature review on efficacy and safety of nonpharmacological treatments. Minerva Pediatr. 2014;66(6):549-57.
19. Garavello W, Somigliana E, Acaia B, Gaini L, Pignataro L, Gaini R. Nasal lavage in pregnant women with seasonal allergic rhinitis: a randomized study. Int Arch Allergy Immunol. 2010;151(2):137-41.

20. Baraniuk J, Petrie K, Le U, Tai C, Park Y, Yuta A, et al. Neuropathology in rhinosinusitis. Am J Respir Crit Care Med. 2005;171(1):5-11.

21. Baraniuk JN, Ali M, Naranch K. Hypertonic saline nasal provocation and acoustic rhinometry. Clin Exp Allergy. 2002;32(4): 543-50.

22. Lenoir J, Bachert C, Remon JP, Adriaens E. The slug mucosal irritation (SMI) assay: a tool for the evaluation of nasal discomfort. Toxicol In Vitro. 2013;27(6):1954-61. https://doi.org/10.1016/j.tiv. 2013.06.018.

23. Rabago D, Zgierska A, Mundt M, Barrett B, Bobula J, Maberry R. Efficacy of daily hypertonic saline nasal irrigation among patients with sinusitis: a randomized controlled trial. J Fam Pract. 2002;51(12):1049-55.

24. Tulupov D, Karpova E, Voropaeva E. A novel view of prophylaxis and treatment of chronic adenoiditis in children. Vestn Otorinolaringol. 2011;1(66.69)

25. Rapiejko P, Jurkiewicz D. The use of hypertonic sea-water solution in patients after surgery of the nose and paranasal sinuses. Otolaryngol Pol. 2010;64(1):20-30.

26. Friedman M, Vidyasagar R, Joseph N. A randomized, prospective, double-blind study on the efficacy of dead sea salt nasal irrigations. Laryngoscope. 2006;116(6):878-82.

27. Garavello W, Di Berardino F, Romagnoli M, Sambataro G, Gaini R. Nasal rinsing with hypertonic solution: an adjunctive treatment for pediatric seasonal allergic rhinoconjunctivitis. Int Arch Allergy Immunol. 2005;137(4):310-4.

28. Rabago D, Pasic T, Zgierska A, Mundt M, Barrett B, Macberry R. The efficacy of hypertonic saline nasal irrigation for chronic sinonasal symptoms. Otolaryngol Head Neck Surg. 2005;133(1): 3-8.

29. Wabnitz D, Wormald P. A blinded, randomized, controlled study on the effect of buffered $0.9 \%$ and $3 \%$ sodium chloride intranasal sprays on ciliary beat frequency. Laryngoscope. 2005;115(5):803-5. 